\title{
Rep and PriA helicase activities prevent RecA from provoking unnecessary recombination during replication fork repair
}

\author{
Akeel A. Mahdi, Carol Buckman, Lynda Harris, and Robert G. Lloyd ${ }^{1}$ \\ Institute of Genetics, University of Nottingham, Queen's Medical Centre, Nottingham NG7 2UH, United Kingdom
}

\begin{abstract}
The rescue of replication forks stalled on the template DNA was investigated using an assay for synthetic lethality that provides a visual readout of cell viability and permits investigation of why certain mutations are lethal when combined. The results presented show that RecA and other recombination proteins are often engaged during replication because RecA is present and provokes recombination rather than because recombination is necessary. This occurs particularly frequently in cells lacking the helicase activities of Rep and PriA. We propose that these two proteins normally limit the loading of RecA on ssDNA regions exposed on the leading strand template of damaged forks, and do so by unwinding the nascent lagging strand, thus facilitating reannealing of the parental strands. Gap closure followed by loading of the DnaB replicative helicase enables synthesis of the leading strand to continue. Without either activity, RecA loads more frequently on the DNA and drives fork reversal, which creates a chickenfoot structure and a requirement for other recombination proteins to re-establish a viable fork. The assay also reveals that stalled transcription complexes are common impediments to fork progression, and that damaged forks often reverse independently of RecA.
\end{abstract}

[Keywords: DNA repair; RuvABC; RecBCD, RecFOR; Holliday junction]

Supplemental material is available at http://www.genesdev.org.

Received February 7, 2006; revised version accepted May 23, 2006.

DNA replication fork complexes are intrinsically processive but often encounter problems that damage the fork and provoke recombination. This recombination may be necessary in some cases to complete replication and maintain viability, but a number of studies have suggested that recombination proteins may also target stalled forks and initiate exchanges when there is no need. They may even cause harm by provoking unequal exchanges between repeated sequences (Rothstein et al. 2000; Fabre et al. 2002; Doe and Whitby 2004; Schmidt and Kolodner 2004). Faithful duplication of the genome may rely therefore not only on the ability to minimize impediments to fork progression and to rescue any forks that have been damaged, but also to limit unnecessary recombination. Failure to do so can be catastrophic for genome stability and cell survival-for instance, it may lead to cancer predisposition and premature aging in humans, as with Bloom's syndrome (Chakraverty and Hickson 1999).

A clear picture of how replication and recombination

${ }^{1}$ Corresponding author.

E-MAIL bob.lloyd@nottingham.ac.uk; FAX 44-115-8230313.

Article is online at http://www.genesdev.org/cgi/doi/10.1101/gad.382306. can become entangled is emerging through studies of how forks are rescued in Escherichia coli (Kowalczykowski 2000). Damaged forks recover via mechanisms that rely ultimately on either PriA or PriC. These two proteins facilitate assembly of the replicative machinery. They target branched DNA, either a fork or a D-loop generated by recombination, and therefore have the potential to initiate replication at any point along the chromosome where such structures arise (Liu and Marians 1999; Marians 2000; Heller and Marians 2005a). They do so by loading the DnaB replicative helicase, the first step in the assembly of a replisome (Fig. 1A). PriA favors forks with a $3^{\prime}$ leading strand hydroxyl at the branch point (Fig. 1C, panel i), a preference also satisfied at D-loops (Fig. 1B [panel ii], C [panel ii]). PriC prefers a fork with a gap in the leading strand, a situation likely to arise when synthesis of the leading strand is blocked while the lagging strand continues to grow (Pages and Fuchs 2003). It also enables DnaG to prime the leading strand de novo, which may allow leading strand synthesis to resume downstream of a blocking lesion, as envisaged for lagging strand synthesis, leaving a gap in the nascent strand (Fig. 1C, panels iii-v; Heller and Marians 2006). 
Mahdi et al.

A


B

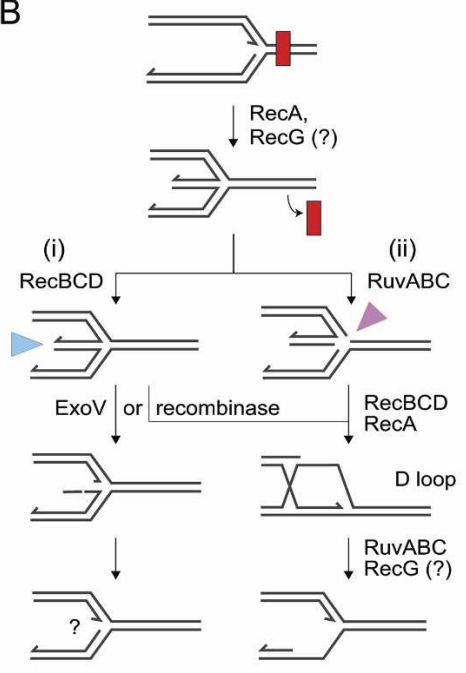

E

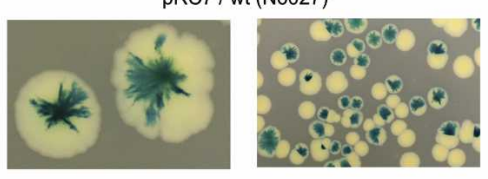

C

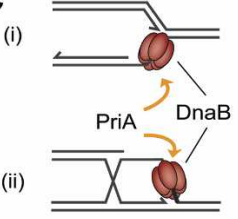

(iii)

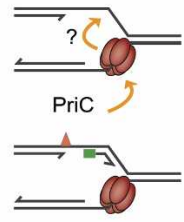

(v)

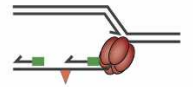

(vi)

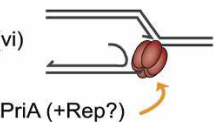

(vii)

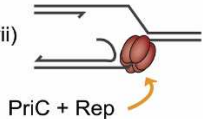

F

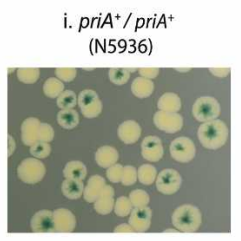

$0.66(672 / 1025)$

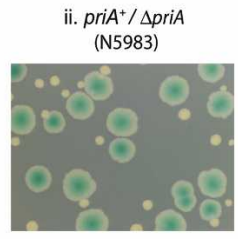

$0.38(578 / 1513)$



$<0.0006(0 / 1555)$

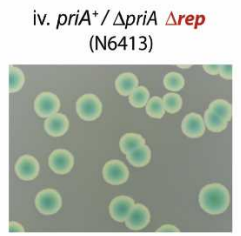

$<0.00073(0 / 1366)$

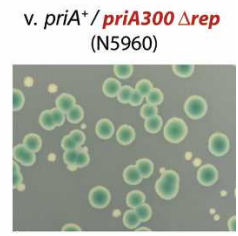

$0.23(392 / 1709)$

Figure 1. Synthetic lethality assay. (A) The E. coli replisome complex, reproduced from McGlynn and Lloyd (2002). (Reprinted with permission from Nature Reviews Molecular Cell Biology. (c) 2002 Macmillan Magazines Ltd. [http://www.nature.com/reviews].) (B) Rescue of a stalled replication fork. Stalling of the fork at a lesion (red rectangle) triggers fork regression. Subsequent processing of the chickenfoot initiated by RecBCD enzyme (panel i) or RuvABC resolvase (panel ii) establishes a fork upstream of the original block. (C) Possible substrates and mechanisms for loading the DnaB hexamer ring on the template for lagging strand synthesis. Green boxes represent RNA primers synthesized by DnaG, and red triangles represent lesions blocking synthesis by Pol III. (D) Structure of pRC7 showing the genetic elements utilized. $(E)$ Segregation of plasmid-free cells in cultures of $\Delta$ lacIZYA strain TB28 carrying pRC7 (strain N6027). (F) Synthetic lethality assays showing the effect of priA, rep, and priC on cell viability. All constructs carry a priA $A^{+}$derivative of pRC7 and the chromosomal mutations indicated. The relevant genotype is shown above each photograph, with the strain number in parentheses. The fraction of white colonies is shown below with the number of white colonies/total colonies analyzed in parentheses.

PriA also has a $3^{\prime}-5^{\prime}$ helicase activity, and may unwind a lagging strand at a fork to make room for DnaB (Zavitz and Marians 1992; McGlynn et al. 1997; Gregg et al. 2002; Cadman et al. 2005). However, Rep helicase has the same polarity and might do the same job (Fig. 1C, panel vi; Heller and Marians 2005b). PriC has no helicase activity but could load DnaB at a fork with a lagging strand with the aid of Rep or PriA (Fig. 1C, panel vii). Genetic studies are consistent with both Rep-PriC and PriA-PriC pathways (Sandler 2000).

Some processing of a stalled fork may be needed to remove or repair any impediment to progression before loading DnaB. RecA recombinase, RecG translocase, or the positive supercoils ahead of the fork may force the branch point back far enough for this to occur, and establish a "chickenfoot" structure that RecBCD, RuvABC, and other recombination proteins may process to restore a fork or form a D-loop (Fig. 1B; Seigneur et al. 1998, 2000; McGlynn and Lloyd 2000; McGlynn et al. 2001; Robu et al. 2001, 2004). However, the extent to which these events are necessary is far from clear. In this report, we show that Rep and PriA helicase activities are key factors in determining whether recombination proteins are brought into play. Without Rep in particular, RecA initiates recombination even though the need to do so may not exist.

\section{Results}

We investigated how recombination may be engaged during replication using a synthetic lethality assay based 
on pRC7 (Fig. 1D), an unstable mini-F plasmid that is rapidly lost (Bernhardt and de Boer 2004). It carries the lac ${ }^{+}$genes, and therefore its loss is revealed in a $\Delta l a c$ background by segregation of $\mathrm{Lac}^{-}$clones. On plates containing the $\beta$-galactosidase indicator, $\mathrm{X}$-gal, these clones form white colonies or white sectors within blue $\left(\mathrm{Lac}^{+}\right)$ colonies (Fig. 1E), depending on whether plasmid loss occurred before or after plating. We made derivatives carrying wild-type alleles of genes of interest to cover a chromosomal mutation before introducing mutations at other, uncovered loci. Synthetic lethality between the covered and uncovered mutations is indicated when plasmid-free segregants fail to grow, with the result that the construct yields uniformly blue colonies (Bernhardt and de Boer 2004).

We first made a $p r i A^{+}$derivative of $\mathrm{pRC} 7$ to cover a deletion $\left(\right.$ priA $^{+} / \Delta$ priA). Cells lacking PriA have reduced viability (Sandler 2000), and so the plasmid should confer a growth advantage. On plating on X-gal, this advantage was demonstrated by the fact that white colonies from plasmid-free segregants are much smaller than blue colonies and also fewer in number than when $p \mathrm{~A}^{+}$is on the chromosome (Fig. 1F, panels i,ii). This construct enabled us to delete priC or rep. Both derivatives yield healthy blue colonies but no white colonies (Fig. 1F, panels iii,iv), indicating that only cells retaining the plasmid can grow.

These data provide compelling visual evidence of the inviability of cells lacking both PriA and PriC, or PriA and Rep (Sandler 2000). They also validate the assay. Thus, the $\mathrm{priA}^{+}$clone segregates as freely as $\mathrm{pRC} 7$ when the chromosome carries priA ${ }^{+}$(Fig. 1F, panel i). Indeed, none of the constructs we made is any more stable than pRC7. Some segregate even more freely, which increases the sensitivity of the assay. Figure 1F, panel ii, shows that when viability is reduced but not abolished, cells carrying the plasmid often form colonies that appear uniformly blue. This is explained by the more robust growth of these cells, which engulfs any white sectors. We also found that the priA $A^{+}$plasmid-in fact, any of the pRC7 derivatives made-may integrate into the chromosome to give stable blue colonies (data not shown). However, this is very rare and produces much deeper blue colonies than those caused by synthetic lethality, which are diluted by abortive growth of plasmid-free cells. Nevertheless, we took care to confirm synthetic lethality by making independent constructs, by combining the mutations in the chromosome under plasmids covering different loci, and by attempting to combine them without any covering plasmid.

\section{Low viability of cells lacking PriA and Rep helicase activities}

The helicase activities of PriA and Rep provide means to unwind a lagging strand at a stalled fork and thus create room for DnaB (Fig. 1C; Heller and Marians 2005a,b). Therefore, cells lacking both activities might find it difficult to restart replication. To test this, we made a priA300 rep strain, covering priA300 with the priA ${ }^{+}$de- rivative of pRC7 before introducing rep. The priA300 allele encodes PriA $_{\mathrm{K} 230 \mathrm{R}}$, which lacks helicase activity but retains the ability to load DnaB (Zavitz and Marians 1992), consistent with priA300 cells lacking the severe defects of priA-null mutants (Sandler 2000; Sandler et al. 2001). The construct yields robust blue colonies and small white colonies (Fig. 1F, panel v), much smaller than those of priA300 and rep controls (Fig. 2G). But, these white colonies can be subcultured. Indeed, a priA300 rep strain can be made without the plasmid (Supplementary Table S1), as reported (Sandler 2000). Broth cultures of such constructs contain reduced numbers of viable cells (Fig. 2A,B) and accumulate suppressors (Fig. 2C), the identities of some of which are revealed later. The cells are elongated and display aberrant nucleoid segregation, or lack DNA (Fig. 2D), but they survive UV irradiation almost as well as rep cells (Fig. 2F). Together, these data demonstrate that the viability of cells lacking PriA and Rep helicases is much reduced, consistent with their having a difficulty in rescuing stalled forks.

\section{Viability is maintained by recombination}

At a D-loop, there is normally no need for a helicase to expose a landing pad for DnaB (Liu and Marians 1999). It is possible this is the only substrate PriA $_{\mathrm{K} 230 \mathrm{R}}$ can use, making fork rescue in priA300 rep cells dependent on RecBCD and RecA to generate a D-loop, and RuvABC to resolve the Holliday junction produced (Fig. 1B). We tested this by combining priA300 $\Delta r e p$ with null alleles of ruv or recB, using pRC7 carrying pri $A^{+}$, rep $^{+}$, or rec $B^{+}$. The constructs yield only blue colonies (Fig. 2G; data not shown), indicating synthetic lethality and consistent with recombination being essential. The effects of knocking out different components of RuvABC are the same, which suggests junction cleavage is required. Controls revealed priA300 ruv and rep ruv cells are viable (Fig. 2G) but much more sensitive to UV light than ruv cells (Fig. 2F). This supports the idea that Rep and PriA helicases can act independently to reduce the need for junction resolution.

We also removed RecD, which abolishes the exonuclease activity (ExoV) of RecBCD but not its recombinase function. It reduces viability (Fig. 3A). We believe this reflects a specific need for a D-loop with an exposed lagging strand template, as would be generated by RecBCDRecA recombination of a reversed fork (Fig. 1B). Without the ExoV activity, the RecBC-RecA reaction might deliver a D-loop with the 5' strand of the initiating duplex paired with the displaced strand (Fig. 3B). Without Rep, and no helicase activity of its own, PriA ${ }_{\mathrm{K} 230 \mathrm{R}}$ would then have difficulty finding a landing pad for DnaB.

RuvABC and RecBCD are essential only when RecA is present

In contrast, a recA-null allele improves growth of priA300 rep cells, and even confers viability on their ruv derivatives (Fig. 3C). Thus, it appears RecA causes the 
Mahdi et al.

A



wt
priA300 priA300
vrep

D

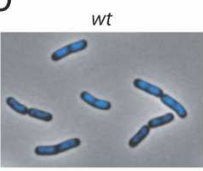

priA300

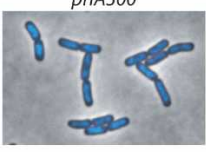

$\Delta r e p$

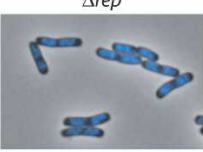

G

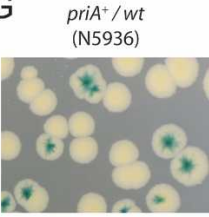

$0.66(672 / 1025)$

$\mathrm{priA}^{+} / \triangle$ ruvaBC (N5955)

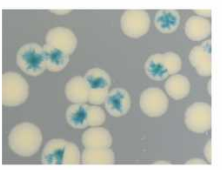

$0.68(849 / 1249)$
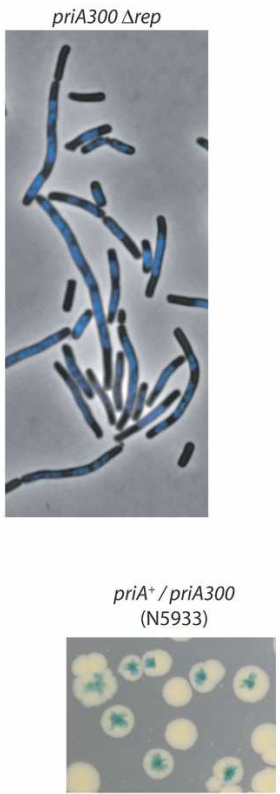

$0.50(1281 / 2534)$

priA $^{+} /$priA300 $\triangle$ ruvABC (N5944)

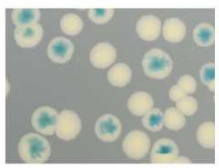

$0.58(426 / 723)$
B

\begin{tabular}{|c|c|c|}
\hline Strain & Genotype & $\mathrm{cfu} / \mathrm{ml}^{-8}$ \\
\hline MG1655 & pri $^{+}$rep $^{+}$ & $1.65 \pm 0.14(n=9)$ \\
\hline N5500 & priA300 & $1.63 \pm 0.24(n=4)$ \\
\hline N5584 & $\Delta r e p$ & $1.18 \pm 0.1(n=8)$ \\
\hline N5587 & priA300 $\Delta$ rep & $0.47 \pm 0.073(n=10$ \\
\hline
\end{tabular}

E

$\square \mathrm{N} 6033$ (wt)

— N6034 (priA300)

- N6035 (rep)

- N6051 (priA300 rep)

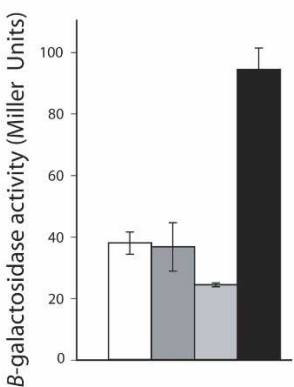

రิ

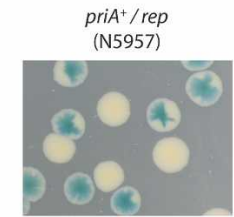

$0.47(564 / 1204)$



$0.53(345 / 647)$



priA300 Arep

F

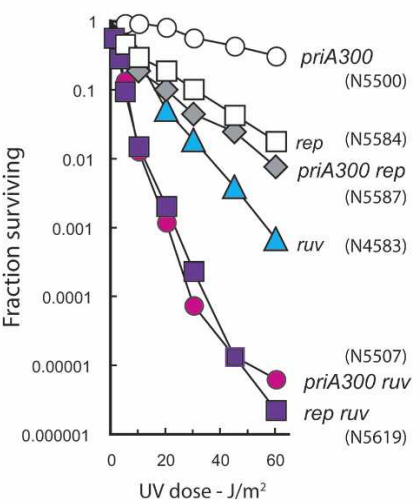

$\mathrm{priA}^{+} /$priA300 rep (N5945)

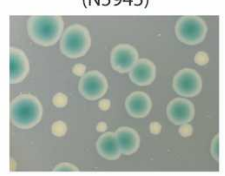

$0.24(686 / 2918)$

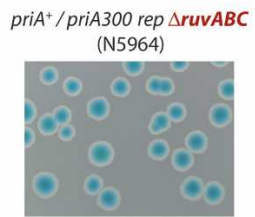

$<0.0009(0 / 1109)$

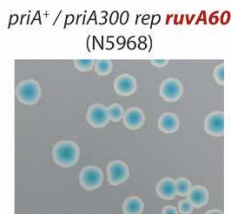

$<0.00067(0 / 1490)$

priA+/priA300 rep $\triangle$ ruvC (N5965)

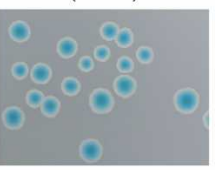

$<0.0025(0 / 396)$

Figure 2. The viability of priA300 rep cells depends on Holliday junction resolution. (A) Growth and viability of priA300 rep cells. Samples from exponential broth cultures of the strains listed in $B$ grown to $A_{650} 0.4$ were diluted and plated as described in Materials and Methods, and photographed after $18 \mathrm{~h}$ at $37^{\circ} \mathrm{C} .(B)$ Viable cell numbers (colony-forming units) in exponential-phase broth cultures grown to $A_{650}$ 0.4. (C) Accumulation of fast-growing variants during subculture of priA300 rep cells. A culture of strain N5587 grown in LB broth from a single colony was incubated overnight, diluted 50-fold in fresh broth, and regrown to saturation before streaking a sample on LB agar. The plate was incubated for $18 \mathrm{~h}$. (D) Effect of priA300 and rep on cell morphology. The strains listed in $B$ were grown to $A_{650} 0.4$ in broth. Merged phase contrast and fluorescence images are shown, displaying Hoechst 33342 fluorescence in cyan. (E) Chronic SOS expression in priA300 rep cells. SOS expression was measured by assaying $\beta$-galactosidase activity in exponential cultures of strains carrying sfiA ::lacZ. (F) Effect of priA300, rep, and ruv mutations on sensitivity to UV light. The strains are listed in parentheses next to the genotype. $(G)$ Synthetic lethality assays illustrating the effect of combining priA300, rep, and ruv mutations. ruvA60 is a Tn10 insertion that inactivates ruvA and prevents transcription of the downstream ruvB.

defects in these cells. We could not test $r e c A$ in the $\mathrm{priA}^{+} /$priA300 rep recD construct because the combination of rep, recD, and recA is lethal (Uzest et al. 1995). Our results show that RuvABC is responsible for this lethality, since we could introduce recA provided we first eliminated RuvABC. The ruv derivative does not segregate white colonies, but the ruv recA derivative does (Fig. 3D), which demonstrates that priA300 rep recD ruv recA cells are viable. These findings reveal that RecA acts before RecBCD and RuvABC, and provokes recombination regardless of the need to do so, thus making RuvABC essential. Two additional lines of investigation support this conclusion.

The RecFOR proteins are required for RecA to have a negative effect

RecA can be loaded directly on single-stranded DNA (ssDNA) exposed by RecBCD recombinase activity. With the aid of the RecF, $\mathrm{RecO}$, and RecR proteins, it may also 
A

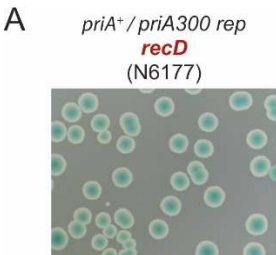

$0.0013(39 / 3057)$

C

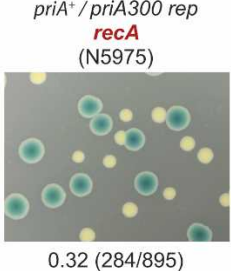

$\mathrm{D}$

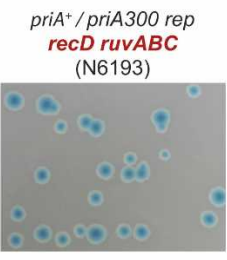

$<0.0011(0 / 913)$

E

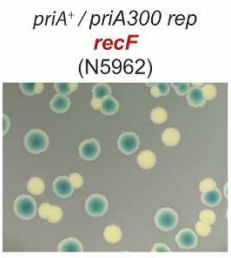

$0.40(246 / 613)$

F

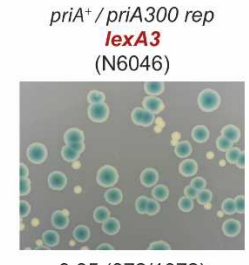

$0.35(372 / 1072)$
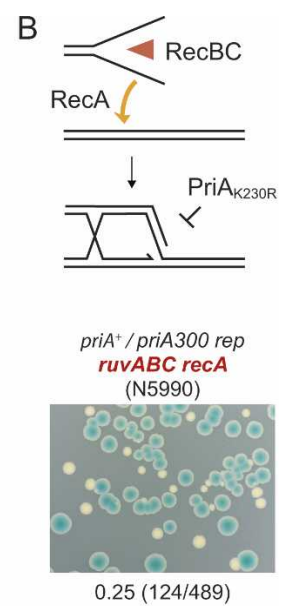

priA / priA300 rep recD ruvABC recA (N6210)

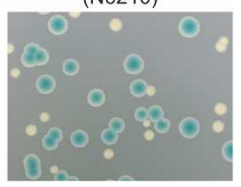

$0.26(150 / 574)$



$0.38(227 / 599)$

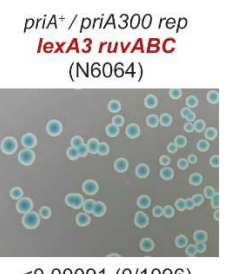

$<0.00091(0 / 1096)$

Figure 3. RecA, RecD, and RecF affect the viability of priA300 rep cells. (A) Synthetic lethality assay illustrating the very low viability of priA300 rep cells lacking ExoV. (B) Recombination without the ExoV activity of RecBCD. The diagram illustrates how recombination initiated at a DNA end via the unwinding activity of RecD-depleted RecBC enzyme (red triangle) might generate an unusual D-loop. $(C-F)$ Synthetic lethality assays illustrating how RecA and RuvABC affect the viability of priA300 rep cells.

be loaded on ssDNA exposed during replication of damaged DNA and coated with SSB protein (Morimatsu and Kowalczykowski 2003). Figure 3E shows that a recF mutation improves growth of priA300 rep cells. This is opposite to the effect of $r e c B$. Furthermore, it removes the need for RuvABC. Essentially identical results were obtained using null alleles of $r e c O$ and $r e c R$. Indeed, mutations at these loci were identified among suppressors of priA300 rep (Fig. 2C; data not shown). These data sug-

gest RecA is loaded on ssDNA exposed during replication in priA300 rep cells.

Such RecA loading would be expected to provoke recombination and also to induce the SOS repair response (Sassanfar and Roberts 1990). Analysis of $\beta$-galactosidase synthesis from lacZ fused to the SOS-induced sfiA gene revealed chronic amplification of SOS in priA300 rep cells (Fig. 2E). This is consistent with the extensive cell filamentation (Fig. 2D). However, preventing SOS induction with a lexA3 mutation does not increase viability, and RuvABC remains essential (Fig. 3F). This contrasts sharply with the effect of recFOR mutations, which also limit SOS induction. Therefore, chronic SOS expression cannot be the major reason why RecA has a negative effect on growth, which leaves RecA recombinase activity as the likely factor.

\section{RecA provokes recombination in the absence of Rep} helicase

To investigate whether RecA drives recombination, we exploited strains deleted for both RecG and RuvC. Such strains lack means to process recombination intermediates, and so exchanges initiated by RecA would be expected to prevent chromosome segregation, and thus reduce viability (Lloyd 1991). We covered a deletion of ruvC with a ruv $C^{+}$derivative of $\mathrm{pRC7}$ before eliminating recG. This construct yields healthy white colonies (Fig. 4A, panel i), which indicates there is insufficient recombination during normal growth to seriously compromise viability. Adding $\Delta r e p$ reduces the yield of white colonies dramatically. The rare examples are tiny and impossible to culture without accumulating suppressors (Fig. $4 \mathrm{~A}$, panel ii). The effect is reversed when $r e c A$ or $r e c F$ is inactivated. In both cases, there is good recovery of very healthy white colonies (Fig. 4A, panels iii,iv). We repeated the studies using pRC7 constructs encoding RecG, RuvAB, or RuvABC to cover mutations eliminating these proteins, with essentially identical findings (data not shown). We conclude that when Rep is missing, RecA is loaded on ssDNA exposed during replication and provokes recombination even though this recombination is not required to maintain viability.

Recent studies have shown how the presence of SSB on the leading strand template of a fork directs Rep to unwind the nascent lagging strand (Heller and Marians 2005b). We suggest Rep specifically targets a damaged fork with no leading strand at the branch point and unwinds the lagging strand, allowing the parental strands to reanneal (Fig. 4B). Without Rep to close the gap efficiently, RecA loads on the leading strand template with the aid of RecFOR and catalyzes homologous pairing and strand exchange with the sister duplex. The fork is driven backward and a chickenfoot structure is established, thus bringing RecBCD, RecA once more, and RuvABC into play.

\section{Replication fork rescue in the absence of Rep}

A strain lacking Rep is quite healthy, and so if it experiences replication fork reversal by RecA, any chicken- 
Mahdi et al.

Figure 4. RecA provokes recombination in cells lacking Rep helicase. (A) Synthetic lethality assays showing dependence on RecF and RecA. The lower panel under panel ii shows a white colony streaked on LB $(30 \mathrm{~h}$ incubation). (B) A model for alternative processing of a stalled fork by Rep and RecA proteins. The diagram depicts how Rep helicase might be directed by SSB protein (green circles) to unwind the lagging strand of a fork with a gap in the leading strand, thus closing the gap. Alternatively, but especially in the absence of Rep, the RecFOR proteins load RecA (blue ovals) on the leading strand template. RecA promotes homologous pairing and strand exchange with the intact sister duplex, driving fork reversal and forming a chickenfoot structure. (C) Possible ways of converting a chickenfoot to a fork (see text for details). $(D-G)$ Synthetic lethality assays demonstrating how the viability of cells lacking Rep helicase is affected by RecA, RecBCD, RuvABC, and RecG.


$0.063(47 / 749)$

$0.40(295 / 738)$



B
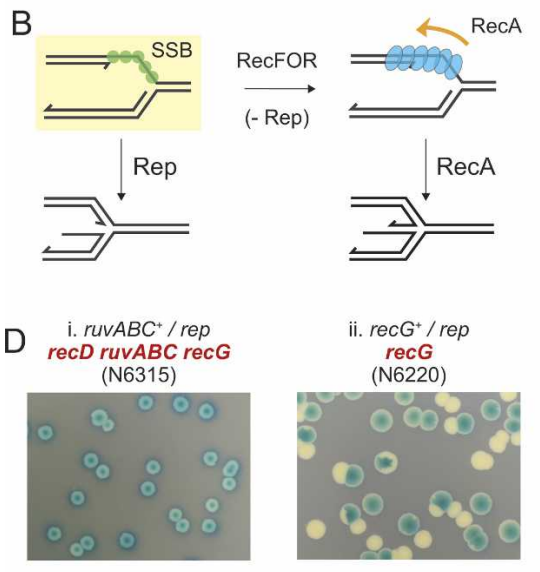

$<0.0014(0 / 708)$



(N6265)

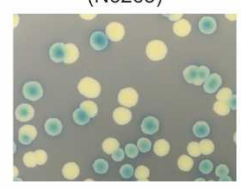

$0.51(218 / 415)$
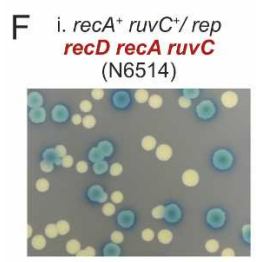

$0.68(390 / 577)$



$0.06(103 / 1714)$
$0.45(445 / 979)$

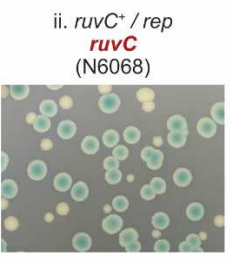

$0.21(179 / 835)$

ii. ruvABC $C^{+} /$rep
recD ruvABC (N6287)



$0.47(564 / 1194)$

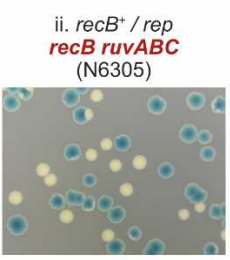

$0.45(510 / 1144)$

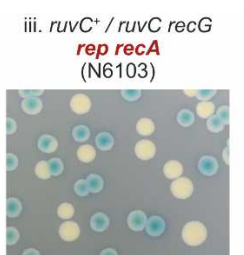

$0.32(356 / 1118)$

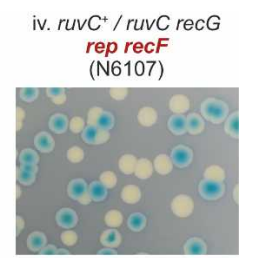

$0.41(288 / 701)$

C
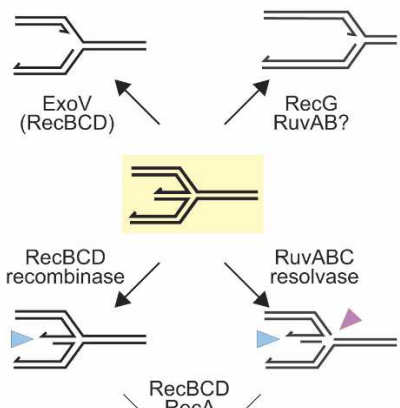

RecA

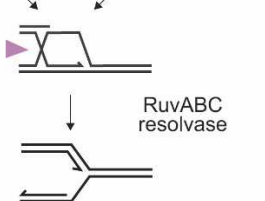

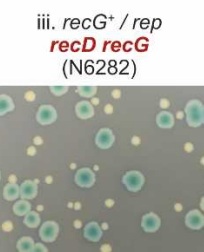

$0.51(327 / 640)$

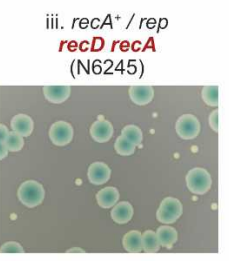

$0.21(212 / 1030)$



$<0.0027(0 / 372)$

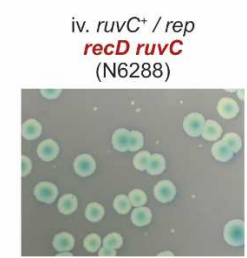

$0.25(123 / 502)$

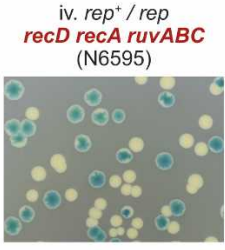

$0.58(413 / 718)$

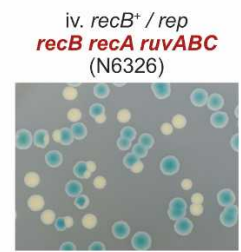

$0.30(204 / 670)$ foot structure formed must be dealt with efficiently. Figure 4C summarizes how this might be achieved. The nascent duplex may be digested by the ExoV activity of RecBCD or recombined via RecBCD-RecA recombinase activities, with or without prior junction cleavage and fork breakage by RuvC, thus generating a fork or D-loop for DnaB loading. Alternatively, branch migration of the junction by RuvAB or RecG may restore the original fork (Fig. 4C). We employed the pRC7-based assay to investigate these possibilities. Our results show that pathways involving duplex DNA digestion or the formation and processing of Holliday junctions are indeed the only ones that can maintain viability. Thus, a rep strain is inviable if RecD and both RuvABC and RecG are elimi- 
nated (Fig. 4D, panel i). The absence of RecD eliminates the few white colonies seen with rep recG ruv cells (Fig. 4A).

The results also indicate that no one activity predominates. Thus, eliminating RecG, ExoV, or RuvC alone causes at most a minor reduction in viability (Fig. 4D [panel ii], E [panels i,ii]). Removing both ExoV and RecG has a greater effect (Fig. 4E, panel iii), indicating that fork rescue is now limited. Removing both ExoV and RuvC is extremely limiting (Fig. 4E, panel iv; data not shown). In this latter case, eliminating RecA restores robust viability (Fig. 4F, panel i), confirming that RecA is responsible for the very low viability. The fact that RuvAB and RecG are present would seem to imply that branch migration is not an effective way to restore a viable fork. However, RuvAB normally forms a complex with RuvC to cleave Holliday junctions, and without it might act aberrantly. Therefore, we analyzed a ruv $A B C^{+} / \triangle \operatorname{ruv} A B C$ rep recD construct. This yields robust white colonies (Fig. 4F, panel ii), but only if RecG is available (Fig. 4D, panel i). Thus, RecG may be able to restore a fork very effectively (Fig. 4C). Its inability to do so in rep recD ruvC cells suggests that RuvAB fails to drive branch migration, or drives unproductive migration. Either way, it blocks RecG.

Our analyses also provided insight into two observations reported previously and that we confirmed during this study. The first is the inviability of rep recD recA cells (Fig. 4F, panel iii; Uzest et al. 1995). Viability is restored if any component of the RuvABC complex is removed (Fig. 4F, panel iv; data not shown), which means that Holliday junction cleavage is responsible for the lethality. Since there is no RecA to drive fork reversal or form Holliday junctions by recombination, this result also reveals that most cells nevertheless suffer fork reversal. With no ExoV, any chickenfoot is likely to persist, increasing the likelihood of junction cleavage and hence fork breakage by RuvABC (Fig. 4C). With no RecA, there is no possibility for rescue by recombination, and the cell is dead. This interpretation is consistent with the reported RuvABC-induced chromosome breakage detected in cells lacking Rep and RecA (Seigneur et al. 2000).

The second observation is that $\operatorname{rep}$ recB cells are inviable, but can be rescued by removing RuvAB or both RuvAB and RuvC, but not RuvC alone (Fig. 4G, panels i,ii; Seigneur et al. 1998; data not shown). The inviability of rep recB cells can be explained by RuvC-mediated fork breakage independently of RecA, as proposed for rep $r e c D$ rec $A$ cells. Indeed, eliminating RecA does not improve the viability of rep recB cells (Fig. 4G, panel iii). It also does not appear to substantially affect the viability of rep recB ruv cells (Fig. 4G, panel iv; data not shown). We assume the reason why removing RuvC alone does not help rep recB cells is because RuvAB blocks RecG, as in rep recD ruvC cells (Fig. 4E, panel iv). It is significant that RuvAB does not similarly interfere in a rep recD recA ruvC cell (Fig. 4F, panel i). Perhaps unwinding of the nascent duplex of the chickenfoot by RecBC dislodges any RuvAB at the junction.

\section{Gap filling at forks}

Recent studies suggest the possibility that both leading and lagging strand synthesis may be discontinuous under certain circumstances, leaving gaps in the nascent strands to be filled in by recombination (Fig. 1C, panels iv,v; Heller and Marians 2006). Our finding that rep recB ruv $A B C$ rec $A$ cells (white colonies) grow despite having no RecA (Fig. 4G, panel iv) implies a majority can replicate the chromosome without leaving a single gap. However, gaps might be formed at forks, but be closed again before replication continues. RecQ and RecJ might close a leading strand gap by unwinding and removing the lagging strand, enabling the parental strands to anneal (Courcelle and Hanawalt 1999). We therefore combined rec $A$ with $r e c Q$ and $r e c /$ mutations in a $\mathrm{pri}^{+} / \mathrm{pri}^{\mathrm{A}} 300$ rep construct to assess whether this would reduce viability. The constructs yield white colonies (Fig. 5A; data not shown). These are as robust as those produced by a pri $A^{+} /$priA300 rep rec $A$ construct (Fig. $3 C$ ). If gaps have to be closed at forks, we suspect they are not closed by RecQ and RecJ. However, they could be closed by a polymerase capable of bypassing any blocking lesion. We used the same type of analysis to test Pol II (PolB), Pol IV (DinB), or Pol V (UmuD'C), all of which have lesion bypass activity (Rangarajan et al. 2002). None is required to maintain the viability of priA300 rep recA cells (Fig. 5B; data not shown). We could not test DNA polymerase I because polA recA cells are inviable (Lloyd et al. 1974). Therefore, we cannot exclude the possibility that gaps are formed but are closed by Pol I in much the same way that Pol I helps to complete lagging strand synthesis.

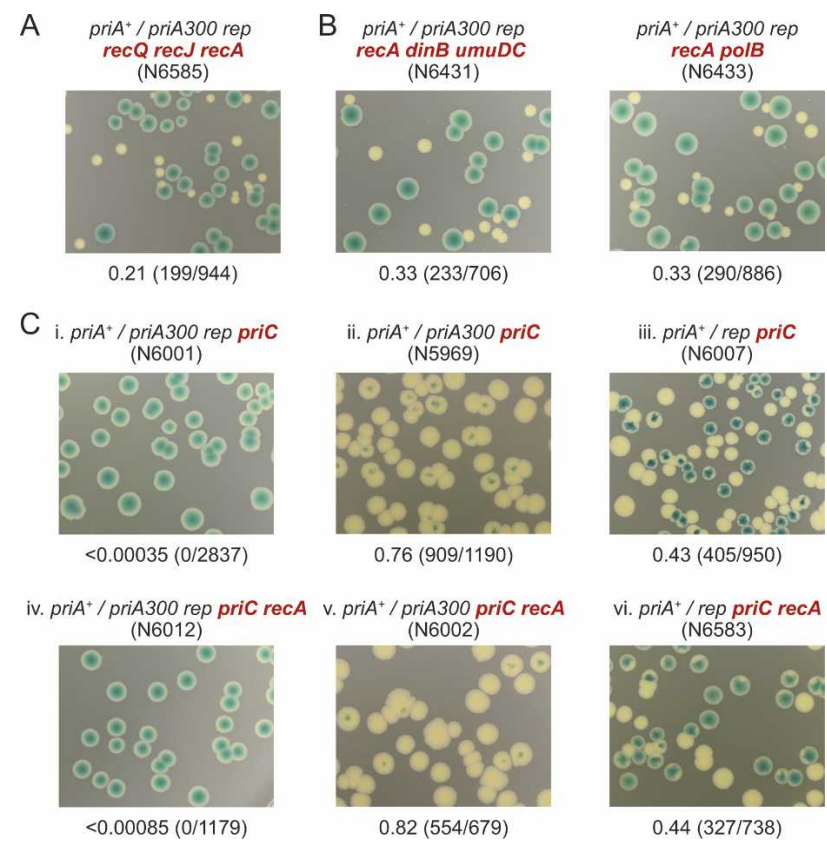

Figure 5. Synthetic lethality assays investigating gap closure and DnaB loading in priA300 rep recA strains. (A) Effect of RecQ helicase and RecJ exonuclease. (B) Effect of DNA polymerases II, IV, and V. (C) Requirement for PriC to load DnaB. 


\section{DnaB loading and gap closure}

The possibility of a gap appearing in the leading strand arises because the PriC system of DnaB loading at a stalled fork may prime synthesis de novo (Heller and Marians 2006). We assumed this would happen rarely in priA300 rep cells because PriC would be ineffective without the helicase activity needed to load DnaB, forcing viability to rely instead on recombination (Figs. 1B, $2 G, 3$ ). To test this, we deleted PriC. Surprisingly, this proved lethal (Fig. 5C, panel i). Given that survival depends on recombination, this finding implies it is PriC that loads DnaB at the D-loop formed, not PriA $\mathrm{K}_{230 R}$. However, PriC would be expected to do so inefficiently because a $3^{\prime}-\mathrm{OH}$ is likely to be present at the branch point (Heller and Marians 2005a). We suggest this is why priA300 rep cells have such low viability. Why PriA $\mathrm{K}_{\mathrm{K} 230 \mathrm{R}}$ is unable to act is not clear.

We also eliminated PriC from priA300 and rep single mutants. The priA300 priC cells proved healthy (Fig. 5C, panel ii), which suggests PriA $_{\mathrm{K} 230 \mathrm{R}}$ is able to load DnaB efficiently when Rep is present. We assume Rep unwinds the lagging strand of any stalled fork to provide a landing pad on which PriA ${ }_{K 230 R}$ can load DnaB. Figure 5C, panel iii shows that rep priC cells are also healthy, as demonstrated previously (Sandler 2000), which is not surprising as they have PriA to load DnaB. Since frequent reversal of a fork would be expected without Rep present, this result also suggests that recombination followed by PriA-mediated loading of DnaB at a D-loop is a very effective way of dealing with a chickenfoot structure.

We deleted RecA to see if recombination is necessary. It made no difference to priA300 rep priC cells (Fig. 5C, panel iv). They remain inviable, which given they have no means to load DnaB is to be expected. However, removing RecA also has no visible effect on the viability of priA300 priC and rep priC cells (Fig. 5C, panels v,vi), which implies that they can replicate their DNA without any need for recombination. Taken together, the results of these assays indicate that by unwinding the lagging strand of a stalled fork and loading DnaB, the combined actions of Rep, PriA, and PriC can rescue forks most of the time without leaving a single gap in the DNA, or at least no gap that needs recombination for its repair.

\section{RNA polymerase (RNAP) complexes are major impediments to fork progression}

The low viability of cells depleted of PriA, and their inviability if also depleted of Rep or PriC (Fig. 1F), implies that replication forks are frequently damaged during normal growth. We exploited the pRC7-based assay to investigate whether this might result from collisions with stalled RNAP complexes, as suggested by Trautinger et al. (2005). We examined strains lacking both GreA and GreB and therefore limited in their ability to reactivate backtracked RNAP complexes efficiently, or lacking DksA and therefore limited in the ability of open complexes to escape certain promoters (Marr and Roberts
2000; Perederina et al. 2004), and asked whether they required recombination for growth. The results revealed that these cells rely on RecBCD, RecA, and PriA to stay alive (Fig. 6A, panels i-vi).

The viability of greA gre $B$ cells is not noticeably affected by eliminating RuvABC or RecG alone (Fig. 6B, panels i,ii), but eliminating both is lethal (Fig. 6B, panel iii), consistent with some means of processing Holliday junctions being essential. However, removing RuvABC enables $r e c B^{+} /$rec $B$ gre $A B$ and $r e c A^{+} /$rec $A$ gre $A B$ constructs to yield robust white colonies (Fig. 6B, panels iv, v). This suggests RuvC-mediated Holliday junction cleavage is responsible for the low viability of gre $A B$ $r e c B$ and gre $A B$ rec $A$ cells. To test this, we replaced RuvABC with RusA resolvase, using a rus-2 insertion to activate the quiescent rusA gene (Mahdi et al. 1996). The construct yields predominantly blue colonies with a small fraction of tiny white colonies (Fig. 6B, panel vi). RusA has no intrinsic branch migration activity and cleaves Holliday junction structures with high specific-


$0.05(52 / 1035)$
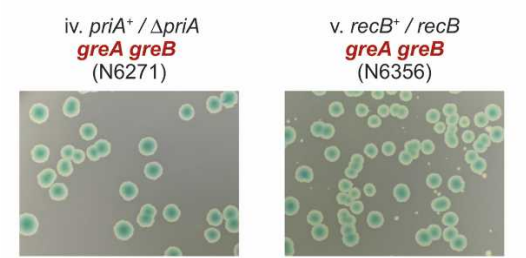

vi. $\operatorname{rec} A^{+} / \operatorname{rec} A$ greA gre $B$
(N6357)

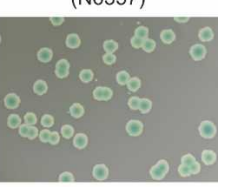

$<0.0012(0 / 823)$

$0.33(297 / 907)$

$0.09(234 / 2362)$

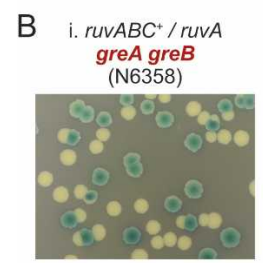

$0.58(435 / 747)$

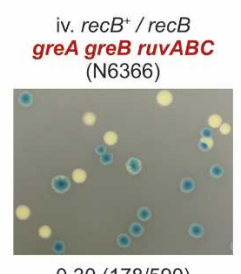

$0.30(178 / 590)$

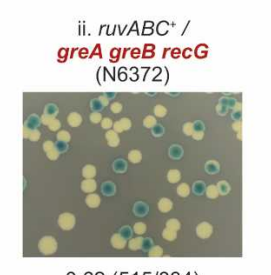

$0.62(515 / 834)$

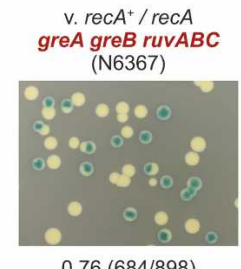

$0.76(684 / 898)$

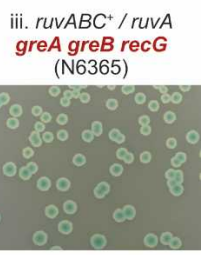

$<0.00062(0 / 1621)$

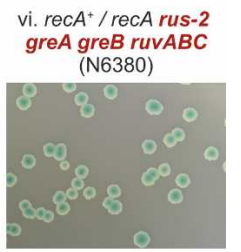

$0.016(24 / 1491)$
Figure 6. Synthetic lethality assays showing how recombination maintains viability in strains with reduced means to minimize the incidence of stalled RNAP complexes. (A) Requirement for recombination in strains lacking DksA, or GreA and GreB. $(B)$ Holliday junction resolution induces recombination in strains lacking GreA and GreB. 
ity (Bolt and Lloyd 2002). Thus, we conclude that viability is reduced as a result of junction cleavage.

These data may be explained if a fork collides with an array of stalled or backtracked RNAP complexes and then reverses to form a chickenfoot. RuvABC cleaves the junction, breaking the fork and forcing recovery via recombination and PriA-mediated loading of DnaB at a Dloop (Fig. 7A, panel i). Without RuvABC to break the fork, RecG may drive the fork back to where it was blocked, enabling PriA to load DnaB without recombination.

This model predicts that reducing the intrinsic stability of RNAP should reduce the number of damaging collisions and therefore the need for recombination. We investigated this using the rpo*35 mutation, which produces an RNAP that forms less stable transcription complexes (Trautinger et al. 2005). Substituting this enzyme for wild-type RNAP improves the growth of $\triangle p r i A$ cells (cf. the relative sizes of blue and white colonies in Figs. 6A [panel ii], 7B [panel i]) and restores viability to priA dksA cells (cf. Figs. 7B [panel ii] and 6A [panel iii]). It also improves growth of priA ruv cells, which are viable but very sick (Fig. 7B, panels iii,iv), as reported pre- viously (Gregg et al. 2002; Grompone et al. 2004b). However, removing RecA leads to an even greater improvement (Fig. 7C, panel i), consistent with recombination being largely responsible for the low viability. Significantly, and regardless of whether RuvABC is present or not, combining $r p o^{*} 35$ and $r e c A$ mutations makes PriA effectively redundant, as there is no obvious growth advantage to be gained from retaining the $p r i A^{+}$plasmid (Fig. 7C, panels ii,iii). This raises the possibility that forks are now rarely damaged.

To test this, we eliminated PriC or Rep so there would be no means to load DnaB. Removing either proved lethal (Fig. 7C, panel iv; data not shown). These data demonstrate that replication forks are still damaged in $r p o^{*}$ cells and have to be rescued. We assume they collapse spontaneously or run into troublesome sequences that uncouple synthesis by the polymerase subunits (Fig. 7A, panel ii). With PriA, RecA, and RuvABC missing, they could not be rescued by recombination. If there is no lesion in the template, there is no reason why the combined action of Rep and PriC should not rescue the fork directly (Fig. 7A, panel ii). Rep could unwind any lagging strand, allowing any gap in the leading strand to be
A



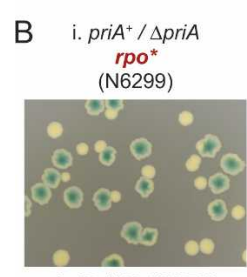

$0.47(1824 / 3870)$

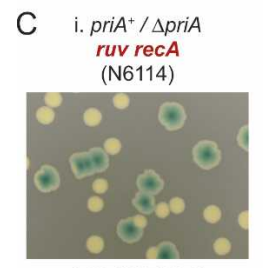

$0.54(265 / 488)$



$0.47(621 / 1331)$

ii. $p r i A^{+} / \triangle p r i A$ rpo* recA (N6321)



$0.71(856 / 1213)$



$0.048(50 / 1051)$

iii. priA $A^{+} / \Delta p r i A$ rpo* ruv recA
(N6344)

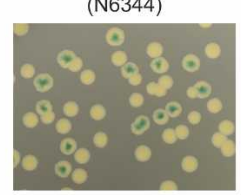

$0.69(897 / 1303)$



$0.33(211 / 643)$

iv. priA $A^{+} / \Delta p r i A$ (N6345)

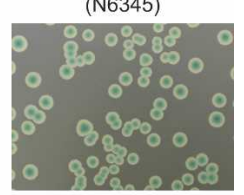

$<0.00044(0 / 2275)$ rpo* ruv recA rep
Figure 7. Interplay between transcription and replication. (A) Models of how RNAP might block replication and provoke recombination. (Panel i) Arrays of stalled or backtracked RNAP complexes (green ovals) accumulate at problematic sites when the cell's ability to destabilize such complexes is compromised as a result of reduced activity of RNAP modulators (ppGpp, DksA, GreA/B, or Mfd). A replication fork may stall at such an array and reverse to form a chickenfoot, providing ways in which RuvABC, RecA, and RecBCD might restore a viable fork. (Panel ii) With a full complement of RNAP modulators, or with $r p o^{*} 35$ present to reduce the intrinsic stability of RNAP, arrays are less prevalent. However, a fork will often stall for other reasons. Spontaneous dissociation of the leading strand polymerase from the leading strand $3^{\prime} \mathrm{tOH}$ might expose the leading strand template. Provided PriA, or Rep and PriC, are present to close the gap and load DnaB, coupled leading and lagging strand synthesis may resume. Without PriA or Rep, there is greater risk of ssDNA remaining exposed. RecFOR may then load RecA (blue ovals), which may drive fork reversal. Alternatively, PriC may load DnaB in a manner that leaves a gap in the nascent leading strand. $(B, C)$ Synthetic lethality assays illustrating how the viability of cells lacking PriA is affected by a mutation $\left(r p o^{*} 35\right)$ reducing the intrinsic stability of RNAP complexes and by the presence of RecA and RuvABC proteins. 
closed by annealing (Fig. 4B), and enabling PriC to load DnaB. If Rep fails to close a gap before RecFOR loads RecA, fork reversal and recombination may be inevitable, explaining why priA single mutants are so sick.

\section{Discussion}

The results presented in this paper reveal rather perversely that RecA and other recombination proteins are often engaged during replication because RecA is present and provokes recombination at damaged forks, and not because recombination is needed to complete chromosome duplication. They also reveal how the helicase activities of Rep and PriA may limit unnecessary deployment of RecA.

\section{Overlapping roles of Rep and PriA helicases}

The discovery that Rep is directed to unwind the nascent lagging strand of a fork by the presence of SSB on the leading strand template (Heller and Marians 2005b) suggests Rep may act to limit the aftermath of replication fork arrest rather than to clear the path for replication as suggested previously (Matson et al. 1994). The data presented in this paper are consistent with this. They indicate that Rep limits the loading of RecA at a damaged fork by facilitating the closure of any leading strand gap (Fig. 4B). Without Rep, RecA loads on the exposed ssDNA and drives fork reversal. Other recombination proteins are then brought to bear on the reversed fork. Thus, many of the features of Rep-deficient cells may reflect more on the pathological consequences of RecA loading than on the frequency with which recombination proteins underpin replication. Fork reversal may occur in the absence of RecA, but less efficiently, making other recombination proteins somewhat redundant.

Our analysis of priA300 rep cells suggests PriA helicase also limits RecA loading. PriA unwinds the lagging strand of a fork and loads DnaB (McGlynn et al. 1997; Gregg et al. 2002). Ironically, the absence of this activity may open the door for RecA to drive fork reversal and thus increase the requirement for PriA-mediated restart, which would explain why priA-null cells are so particularly sick and why eliminating RecA makes a significant improvement. Without RecA to drive reversal, the requirement for PriA is reduced.

The DNA-unwinding activity of Rep and PriA, coupled with timely loading of DnaB to restart replication, may be necessary to counter the fact that RecA is an abundant protein designed to bind ssDNA and promote strand exchange. A high basal level of RecA may ensure efficient recombination and repair of damaged DNA, but brings with it the danger that RecA may be loaded at replication forks whenever ssDNA is exposed. The replisome complex has probably evolved to avoid RecA loading on the unwound but still unreplicated lagging strand template (Fig. 1A). However, specific mechanisms may have to be employed to limit loading on the leading strand template whenever synthesis of the na- scent leading strand is disrupted. The timely action of Rep and PriA in the manner we have proposed would have this effect. It would be consistent with the observation made several times during the course of this work that inactivating RecA improves viability, which is not what would be expected if recombination vitally underpins replication.

Eukaryotic cells deploy several helicases (e.g., Sgs1, Srs2, Rrm3, Fbh1) to limit or counter reversal of stalled forks and avoid unnecessary mitotic recombination by Rad51, the eukaryotic homolog of RecA (Fabre et al. 2002; Schmidt and Kolodner 2004; Osman et al. 2005). Srs2 has been shown to remove Rad51 assembled on ssDNA rather than to prevent Rad51 assembly (Krejci et al. 2003; Veaute et al. 2003). Some of our results could be explained if Rep were to act in a similar fashion. Recent studies suggest otherwise, revealing instead that RecA is displaced by UvrD helicase (Veaute et al. 2005). Our suggestion that Rep limits RecA activity is therefore consistent with the extremely poor viability of rep uvrD cells, which may be a consequence of RecA loading by RecFOR (Petit and Ehrlich 2002; Veaute et al. 2005). However, there is also evidence $\mathrm{UvrD}$ may promote fork reversal and subsequent chromosome breakage by RuvABC under certain conditions (Flores et al. 2004). Thus, by removing RecA from ssDNA exposed at stalled forks, UvrD may also allow other factors to drive fork reversal (Flores et al. 2004).

\section{Fork stalling and post-replication repair}

The idea that recombination proteins can be brought into play unnecessarily does not belie the fact that there are occasions when their activity is vital. Indeed, our analysis of how viability is affected by RNAP modulators suggests they often have to deal with the aftermath of collisions between replication forks and stalled transcription complexes. Recombination is also needed to repair broken forks and close any gaps left in the daughter duplexes. At first sight, the fact that $>60 \%$ of cells in cultures of $r e c A$ mutants are viable would suggest events leading to breaks or gaps do not occur every cell cycle. However, the high viability may be misleading if RecA causes some of these events.

RecA-mediated recombination is clearly important for the survival of UV-irradiated cells. Indeed, all the cells lacking RecA, RecBCD, or RuvABC we studied are sensitive to UV (Fig. 2F; results not presented). In the classical Rupp and Howard-Flanders model, replication forks proceed in UV-irradiated cells, leaving gaps in the daughter duplexes to be filled in by strand exchange (Fig. 1C, panels iv,v; Rupp et al. 1971). Analysis of the DNA made immediately after irradiation reveals the nascent strands are initially of low molecular weight, but increase to match the bulk chromosomal DNA within 30-60 min post-irradiation incubation, consistent with the model. However, Khidhir et al. (1985) showed most of this DNA synthesis depends on DnaA, which suggests it is made by new forks assembled at oriC. The pre-existing forks do recover provided RecA is available, but may take up 
to $90 \mathrm{~min}$ to complete replication (Khidhir et al. 1985), indicating they stall and undergo extensive processing before restarting synthesis. Therefore, measures of net DNA synthesis after UV irradiation need to be adjusted for origin firing before any effect on existing fork progression can be assessed.

The data presented here and elsewhere (Seigneur et al. 1998, 2000; Flores et al. 2001; Grompone et al. 2004a,b) strongly support the view that arrested forks undergo extensive processing by recombination proteins before DNA replication can restart. Admittedly, these studies have examined unirradiated cells, but fork progression may face similar problems as a result of endogenous DNA damage or encounters with troublesome sequences or transcription complexes.

A likely scenario for UV-irradiated cells is that the leading strand template is exposed when a fork encounters a lesion blocking synthesis by the leading strand polymerase. Because leading and lagging strand synthesis are normally coupled, fork progression is brought to a halt (Khidhir et al. 1985). With no immediate means to extend the leading strand past the noncoding UV lesion, gap closure via Rep and PriA helicase activities may be futile, with the result that RecA is brought into play. RecA loading, SOS induction, fork reversal, and processing of the chickenfoot by RecBCD, RuvABC, and RecG would provide means and time for the UvrABC excision repair proteins to remove the blocking lesion, and most lesions remaining downstream, thus clearing the way for chromosome replication to be completed without further hindrance. Given the first lesion encountered will be in the lagging strand template $50 \%$ of the time, and DnaG enables lagging strand synthesis to resume downstream, the cell may also have to rely on RecA to close the resulting lagging strand gap as in the Rupp and Howard-Flanders model. If DnaG occasionally primes leading strand synthesis in the same way, the need for gap repair may be increased (Heller and Marians 2006). However, the viability of cells lacking PriA, RecA, and RuvABC, and their dependence on PriC (Fig. 7C), indicate that de novo priming of the leading strand is not a common event during normal growth, consistent with the textbook model of replication, or leaves gaps that can be closed without recombination.

\section{Replication fork breakage}

A number of studies have indicated that RuvABC may induce replication fork breakage when replication forks arrest. However, substantial breakage is usually detected only when the exonuclease activity of RecBCD is missing (Seigneur et al. 1998, 2000). This indicates that RecBCD normally acts before RuvABC. Consistent with this, some of the constructs we analyzed revealed a requirement for the ExoV activity of RecBCD to maintain viability (Figs. 3, 4; results not presented). This is certainly true of rep recA strains, but only because RuvABC is present. Without RuvABC, neither ExoV nor indeed RecBCD is required (Fig. 4F,G). However, we also found that the Holliday junction cleavage by RuvABC confers inviability on cells lacking both RecA and the transcription factors GreA and GreB (Fig. 6). This observation implies not only that Holliday junctions are formed in these cells despite the absence of RecA but also that these junctions are targeted and cleaved by RuvABC despite the presence of RecBCD. Taken together, these and other results we have presented indicate that measures of DNA breakage in recA strains may be misleading given RecA itself can be such a strong driver of fork reversal.

There is no denying that RecBCD is likely to target the nascent duplex of a reversed fork and that its absence increases the opportunity for RuvABC to break the fork. Whether its ExoV activity alone can efficiently re-establish a viable fork for replication restart is less clear. The motor activity of RecBCD is extremely powerful (Spies et al. 2003) and might not stop when it reaches the branch point of a reversed fork. Indeed, it may drive further reversal until an encounter with a Chi sequence triggers recombinase activity (Singleton et al. 2004). Thus, RecBCD may usually reset a fork via recombination. Without RecA, the ExoV activity might establish a viable fork, but then without RecA driving fork reversal, there might be less need to do so.

\section{Materials and methods}

\section{Strains and plasmids}

Bacterial strains are listed in Supplementary Table S1. All constructs used for the synthetic lethality assay are derivatives of $E$. coli K-12 MG1655 DlacIZYA (Bernhardt and de Boer 2004) carrying pRC7 or one of its derivatives. Chromosomal genes were inactivated in these constructs using deletion alleles or $\operatorname{Tn} 10$ insertions. pRC7 (Fig. 1D) is a low-copy-number, mini-F derivative of pFZY1 (Bernhardt and de Boer 2004). Wild-type priA, rec, ruv, and rep genes were inserted either at the multiple cloning site (MCS) in the lac promoter or at the ApaI sites within lacI ${ }^{q}$, as described in the Supplemental Material.

\section{Media and general methods}

Growth media and methods for monitoring cell growth, P1vir transduction, determining sensitivity to UV and mitomycin C, and measuring SOS induction in sfiA::1acZ fusion strains have been cited (McGlynn and Lloyd 2000; Trautinger et al. 2005). The number of colony-forming units (cfu) in LB broth cultures grown to mid-exponential phase $\left(A_{650} 0.4\right)$ was determined by spotting $10-\mu \mathrm{L}$ aliquots of serial 10 -fold dilutions, from $10^{-2}$ to $10^{-5}$, on LB agar and incubating for $18 \mathrm{~h}$ at $37^{\circ} \mathrm{C}$ before photographing the plates and counting colonies.

\section{Synthetic lethality assay}

Stock cultures of strains carrying pRC7 or one of its derivatives were diluted 80-fold in LB broth and grown with no ampicillin selection to $A_{650} 0.4$ before plating dilutions on LB agar supplemented with X-gal and IPTG. Plates were photographed and scored after $48 \mathrm{~h}$ of incubation. Unless stated otherwise, white colonies formed by plasmid-free cells could be subcultured on LB agar.

\section{Microscopy}

Cultures were grown in broth to $A_{650} 0.4$, stained with Hoechst 33342 , and immobilized on slides coated with agarose. Fluores- 
cence and phase contrast images were captured using an Olympus BX51 microscope.

\section{Acknowledgments}

We thank Piet de Boer and Tom Bernhardt for advice on the use of pRC7, and the many other colleagues who provided strains. We also thank Jing Zhang for constructing pJJ100, and Christian Rudolph and Tim Moore for help with microscopy. This work was supported by the Medical Research Council.

\section{References}

Bernhardt, T.G. and de Boer, P.A. 2004. Screening for synthetic lethal mutants in Escherichia coli and identification of EnvC $(\mathrm{YibP})$ as a periplasmic septal ring factor with murein hydrolase activity. Mol. Microbiol. 52: 1255-1269.

Bolt, E.L. and Lloyd, R.G. 2002. Substrate specificity of RusA resolvase reveals the DNA structures targeted by RuvAB and RecG in vivo. Mol. Cell 10: 187-198.

Cadman, C.J., Lopper, M., Moon, P.B., Keck, J.L., and McGlynn, P. 2005. PriB stimulates PriA helicase via an interaction with single-stranded DNA. J. Biol. Chem. 280: 39693-39700.

Chakraverty, R.K. and Hickson, I.D. 1999. Defending genome integrity during DNA replication: A proposed role for RecQ family helicases. Bioessays 21: 286-294.

Courcelle, J. and Hanawalt, P.C. 1999. RecQ and RecJ process blocked replication forks prior to the resumption of replication in UV-irradiated Escherichia coli. Mol. Gen. Genet. 262: $543-551$.

Doe, C.L. and Whitby, M.C. 2004. The involvement of Srs2 in post-replication repair and homologous recombination in fission yeast. Nucleic Acids Res. 32: 1480-1491.

Fabre, F., Chan, A., Heyer, W.D., and Gangloff, S. 2002. Alternate pathways involving Sgs1/Top3, Mus81/Mms4, and Srs2 prevent formation of toxic recombination intermediates from single-stranded gaps created by DNA replication. Proc. Natl. Acad. Sci. 99: 16887-16892.

Flores, M.J., Bierne, H., Ehrlich, S.D., and Michel, B. 2001. Impairment of lagging strand synthesis triggers the formation of a RuvABC substrate at replication forks. EMBO J. 20: 619-629.

Flores, M.J., Bidnenko, V., and Michel, B. 2004. The DNA repair helicase UvrD is essential for replication fork reversal in replication mutants. EMBO Rep. 5: 983-988.

Gregg, A.V., McGlynn, P., Jaktaji, R.P., and Lloyd, R.G. 2002. Direct rescue of stalled DNA replication forks via the combined action of PriA and RecG helicase activities. Mol. Cell 9: 241-251.

Grompone, G., Ehrlich, D., and Michel, B. 2004a. Cells defective for replication restart undergo replication fork reversal. EMBO Rep. 5: 607-612.

Grompone, G., Sanchez, N., Dusko Ehrlich, S., and Michel, B. 2004b. Requirement for RecFOR-mediated recombination in priA mutant. Mol. Microbiol. 52: 551-562.

Heller, R.C. and Marians, K.J. 2005a. The disposition of nascent strands at stalled replication forks dictates the pathway of replisome loading during restart. Mol. Cell 17: 733-743.

- 2005b. Unwinding of the nascent lagging strand by Rep and PriA enables the direct restart of stalled replication forks. J. Biol. Chem. 280: 34143-34151.

- 2006. Replication fork reactivation downstream of a blocked nascent leading strand. Nature 439: 557-562.

Khidhir, M.A., Casaregola, S., and Holland, I.B. 1985. Mecha- nism of transient inhibition of DNA synthesis in ultravioletirradiated E. coli: Inhibition is independent of recA whilst recovery requires RecA protein itself and an additional, inducible SOS function. Mol. Gen. Genet. 199: 133-140.

Kowalczykowski, S.C. 2000. Initiation of genetic recombination and recombination-dependent replication. Trends Biochem. Sci. 25: 156-165.

Krejci, L., Van Komen, S., Li, Y., Villemain, J., Reddy, M.S., Klein, H., Ellenberger, T., and Sung, P. 2003. DNA helicase Srs2 disrupts the Rad51 presynaptic filament. Nature 423: 305-309.

Liu, J. and Marians, K.J. 1999. PriA-directed assembly of a primosome on D loop DNA. J. Biol. Chem. 274: 25033-25041.

Lloyd, R.G. 1991. Conjugational recombination in resolvasedeficient ruvC mutants of Escherichia coli K-12 depends on recG. J. Bacteriol. 173: 5414-5418.

Lloyd, R.G., Low, K.B., Godson, G.N., and Birge, E.A. 1974. Isolation and characterization of an Escherichia coli K-12 mutant with a temperature-sensitive RecA- phenotype. I. Bacteriol. 120: 407-415.

Mahdi, A.A., Sharples, G.J., Mandal, T.N., and Lloyd, R.G. 1996. Holliday junction resolvases encoded by homologous rusA genes in Escherichia coli K-12 and phage 82. J. Mol. Biol. 257: 561-573.

Marians, K.J. 2000. Replication and recombination intersect. Curr. Opin. Genet. Dev. 10: 151-156.

Marr, M.T. and Roberts, J.W. 2000. Function of transcription cleavage factors GreA and $\mathrm{GreB}$ at a regulatory pause site. Mol. Cell 6: 1275-1285.

Matson, S.W., Bean, D.W., and George, J.W. 1994. DNA helicases: Enzymes with essential roles in all aspects of DNA metabolism. Bioessays 16: 13-22.

McGlynn, P. and Lloyd, R.G. 2000. Modulation of RNA polymerase by (p)ppGpp reveals a RecG-dependent mechanism for replication fork progression. Cell 101: 35-45.

- 2002. Recombinational repair and restart of damaged replication forks. Nat. Rev. Mol. Cell Biol. 3: 859-870.

McGlynn, P., Al-Deib, A.A., Liu, J., Marians, K.J., and Lloyd, R.G. 1997. The DNA replication protein PriA and the recombination protein RecG bind D-loops. J. Mol. Biol. 270: 212-221.

McGlynn, P., Lloyd, R.G., and Marians, K.J. 2001. Formation of Holliday junctions by regression of stalled replication forks: RecG stimulates fork regression even when the DNA is negatively supercoiled. Proc. Nat1. Acad. Sci. 98: 8235-8240.

Morimatsu, K. and Kowalczykowski, S.C. 2003. RecFOR proteins load RecA protein onto gapped DNA to accelerate DNA strand exchange: A universal step of recombinational repair. Mol. Cell 11: 1337-1347.

Osman, F., Dixon, J., Barr, A.R., and Whitby, M.C. 2005. The F-Box DNA helicase Fbh1 prevents Rhp51-dependent recombination without mediator proteins. Mol. Cell. Biol. 25: 8084-8096.

Pages, V. and Fuchs, R.P. 2003. Uncoupling of leading- and lagging-strand DNA replication during lesion bypass in vivo. Science 300: 1300-1303.

Perederina, A., Svetlov, V., Vassylyeva, M.N., Tahirov, T.H., Yokoyama, S., Artsimovitch, I., and Vassylyev, D.G. 2004. Regulation through the secondary channel-structural framework for ppGpp-DksA synergism during transcription. Cell 118: 297-309.

Petit, M.A. and Ehrlich, D. 2002. Essential bacterial helicases that counteract the toxicity of recombination proteins. EMBO J. 21: 3137-3147.

Rangarajan, S., Woodgate, R., and Goodman, M.F. 2002. Replication restart in UV-irradiated Escherichia coli involving 
pols II, III, V, PriA, RecA, and RecFOR proteins. Mol. Microbiol. 43: 617-628.

Robu, M.E., Inman, R.B., and Cox, M.M. 2001. RecA protein promotes the regression of stalled replication forks in vitro. Proc. Natl. Acad. Sci. 98: 8211-8218.

- 2004. Situational repair of replication forks: Roles of RecG and RecA proteins. J. Biol. Chem. 279: 10973-10981.

Rothstein, R., Michel, B., and Gangloff, S. 2000. Replication fork pausing and recombination, or 'gimme a break.' Genes \& Dev. 14: 1-10.

Rupp, W.D., Wilde, C.E., Reno, D.L., and Howard-Flanders, P. 1971. Exchanges between DNA strands in ultraviolet-irradiated Escherichia coli. J. Mol. Biol. 61: 25-44.

Sandler, S.J. 2000. Multiple genetic pathways for restarting DNA replication forks in Escherichia coli K-12. Genetics 155: 487-497.

Sandler, S.J., McCool, J.D., Do, T.T., and Johansen, R.U. 2001. PriA mutations that affect PriA-PriC function during replication restart. Mol. Microbiol. 41: 697-704.

Sassanfar, M. and Roberts, J.W. 1990. Nature of the SOS-inducing signal in Escherichia coli. The involvement of DNA replication. J. Mol. Biol. 212: 79-96.

Schmidt, K.H. and Kolodner, R.D. 2004. Requirement of Rrm3 helicase for repair of spontaneous DNA lesions in cells lacking Srs2 or Sgs1 helicase. Mol. Cell. Biol. 24: 3213-3226.

Seigneur, M., Bidnenko, V., Ehrlich, S.D., and Michel, B. 1998. RuvAB acts at arrested replication forks. Cell 95: 419-430.

Seigneur, M., Ehrlich, S.D., and Michel, B. 2000. RuvABC-dependent double-strand breaks in dnaBts mutants require RecA. Mol. Microbiol. 38: 565-574.

Singleton, M.R., Dillingham, M.S., Gaudier, M., Kowalczykowski, S.C., and Wigley, D.B. 2004. Crystal structure of RecBCD enzyme reveals a machine for processing DNA breaks. Nature 432: 187-193.

Spies, M., Bianco, P.R., Dillingham, M.S., Handa, N., Baskin, R.J., and Kowalczykowski, S.C. 2003. A molecular throttle: The recombination hotspot chi controls DNA translocation by the RecBCD helicase. Cell 114: 647-654.

Trautinger, B.W., Jaktaji, R.P., Rusakova, E., and Lloyd, R.G. 2005. RNA polymerase modulators and DNA repair activities resolve conflicts between DNA replication and transcription. Mol. Cell 19: 247-258.

Uzest, M., Ehrlich, S.D., and Michel, B. 1995. Lethality of rep recB and rep recC double mutants of Escherichia coli. Mol. Microbiol. 17: 1177-1188.

Veaute, X., Jeusset, J., Soustelle, C., Kowalczykowski, S.C., Le Cam, E., and Fabre, F. 2003. The Srs2 helicase prevents recombination by disrupting Rad51 nucleoprotein filaments. Nature 423: 309-312.

Veaute, X., Delmas, S., Selva, M., Jeusset, J., Le Cam, E., Matic, I., Fabre, F., and Petit, M.A. 2005. UvrD helicase, unlike Rep helicase, dismantles RecA nucleoprotein filaments in Escherichia coli. EMBO J. 24: 180-189.

Zavitz, K.H. and Marians, K.J. 1992. ATPase-deficient mutants of the Escherichia coli DNA replication protein PriA are capable of catalyzing the assembly of active primosomes. J. Biol. Chem. 267: 6933-6940. 


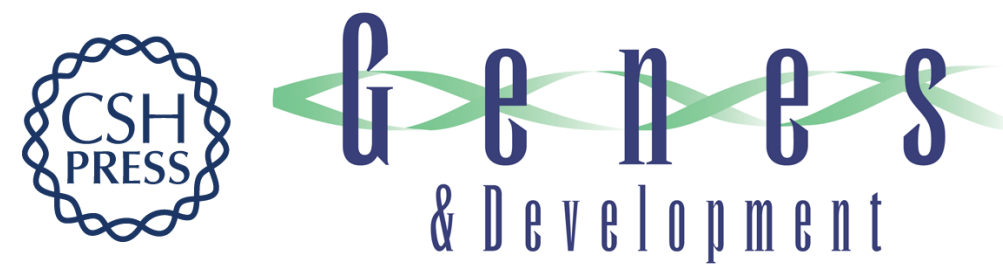

\section{Rep and PriA helicase activities prevent RecA from provoking unnecessary recombination during replication fork repair}

Akeel A. Mahdi, Carol Buckman, Lynda Harris, et al.

Genes Dev. 2006, 20:

Access the most recent version at doi:10.1101/gad.382306

Supplemental http://genesdev.cshlp.org/content/suppl/2006/07/14/20.15.2135.DC1
Material

References This article cites 52 articles, 16 of which can be accessed free at:

http://genesdev.cshlp.org/content/20/15/2135.full.html\#ref-list-1

License

Email Alerting Receive free email alerts when new articles cite this article - sign up in the box at the top

Service

right corner of the article or click here.



